\title{
Electronic Structures and Magnetic Properties of Co-Adsorbed Monolayer $\mathbf{W S}_{2}$
}

\author{
Weiyun Xu \\ School of Materials Engineering, Shanghai University of Engineering Science, Shanghai, China \\ Email:xuweiyun@163.com
}

How to cite this paper: $\mathrm{Xu}, \mathrm{W} . \mathrm{Y} .(2016)$ Electronic Structures and Magnetic Properties of Co-Adsorbed Monolayer $\mathrm{WS}_{2}$. Journal of Materials Science and Chemical Engineering, 4, 32-41.

http://dx.doi.org/10.4236/msce.2016.410004

Received: September 26, 2016

Accepted: October 11, 2016

Published: October 14, 2016

Copyright $\odot 2016$ by author and Scientific Research Publishing Inc. This work is licensed under the Creative Commons Attribution International License (CC BY 4.0).

http://creativecommons.org/licenses/by/4.0/ (c) (i) Open Access

\begin{abstract}
Using the first-principles density functional theory (DFT) calculations, we study the effects of Co adatom on the electronic and magnetic properties of monolayer $\mathrm{WS}_{2}$. The calculations show that, for the high symmetry adsorption sites (Tw, $\mathrm{H}$ and $\mathrm{Ts}$ ) on the surface of monolayer $\mathrm{WS}_{2}$, Co atom prefers $\mathrm{Tw}$ site. The p-d hybridization mechanism for the magnetism results in the splitting of the energy levels near the Fermi energy. A total magnetic moment of $\sim 1.0 \mu_{\mathrm{B}}$ is found in $\mathrm{WS}_{2}$ system with one Co adsorbed and local magnetic moment which mainly focuses on the adsorption site. For Tw adsorption position, we further investigate the formation energy of the ferromagnetic (FM) and the antiferromagnetic (AFM) states under different monolayer coverage (ML) of Co atoms. The FM configurations are relatively stable at 0.50 ML and 1.0 ML. The local density of states (LDOS) and band calculations reveal that both of them present half-metal ferromagnetic materials' property, which are the important preparation materials for spintronic devices.
\end{abstract}

\section{Keywords}

First-Principles, Monolayer $\mathrm{WS}_{2}$, Monolayer Coverage (ML), Half-Metal

\section{Introduction}

Recently, two-dimensional transition metal dichalcogenides (TMDCs), such as $\mathrm{MoS}_{2}$, $\mathrm{WS}_{2}, \mathrm{MoSe}_{2}$ and others, have been extensively studied due to their analogous structure with the best known material of this kind, graphene [1]-[3]. They are expected to have unique electronic properties varying from metal to wide-gap semiconductor [4] [5]. Some TMDCs have been proposed and investigated for possible applications, including transistors [6], photovoltaics [7], catalysis [8], hydrogen storage [9] and Li-ion batteries [10] [11]. Recently, great attention has been paid to explore the magnetic properties for the nanoscale spintronic applications by doping foreign atoms [12]-[16]. The electronic 
structure of the system can be altered, which could lead to appealing character. For instance, a systematic DFT investigation of the absorbed $\mathrm{MoS}_{2}$ monolayer with various atoms, including alkali metals, alkaline earth metals, main group metal, 3d-transition metals, noble metal and nonmetal atoms was performed. Metallic, semimetallic or semiconducting behavior can be found in direct bandgap monolayer $\mathrm{MoS}_{2}$ [17] [18]. In particular, the doping of transition metal atoms (TM) from the IIIB to VIB groups has been confirmed to induce magnetism in nonmagnetic nanomaterials [19] [20]. Mndoped $\mathrm{MoS}_{2}$ monolayers had been demonstrated to be the potential for engineering a new class of atomically thin dilute magnetic semiconductors [21].

As one of the typical TMDs, monolayer $\mathrm{WS}_{2}$, is composed of covalently bonded S-W-S with atomic thickness of $\sim 0.7 \mathrm{~nm}$ and is weakly bonded together by Van der Waals force forming the crystal [22] [23]. Bulk $\mathrm{WS}_{2}$ is an indirect gap semiconductor with a gap of $1.3 \mathrm{eV}$, while the gap becomes direct with size of $\sim 2 \mathrm{eV}$ for a single layer. The layers $\mathrm{WS}_{2}$ have a $\mathrm{P}_{3} / \mathrm{mmc}$ space group symmetry with the $\mathrm{W}$ atoms having a trigonal prismatic coordination with the S atoms [2] [24], which are similar to $\mathrm{MoS}_{2}$ structurally and electronically, as both of them reside in the same column of the periodic table. But $\mathrm{WS}_{2}$ has superior thermal and oxidative stability than that of $\mathrm{MoS}_{2}$. Recent studies show that the monolayers $\mathrm{WS}_{2}$ possess a high in-plane carrier mobility and electrostatic modulation of conductance [25]. The doping of other metallic elements into the monolayer $\mathrm{WS}_{2}$ has been scarcely studied. For example, the Mn-doped $\mathrm{WS}_{2}$ monolayer is found to have a ferromagnetic coupling by a double-exchange mechanism [26] and the properties of $\mathrm{V}, \mathrm{Nb}$ and Ta substituted $\mathrm{WS}_{2}$ monolayers had been reported under S-rich condition [27]. As a matter of fact, a pristine monolayer $\mathrm{WS}_{2}$ is nonmagnetic; for the low-dimensional systems, atom adsorption is an effective doping way to modify its magnetic and electronic properties [28] [29]. In this paper, we investigated the electronic and magnetic properties of Co-adsorbed monolayer $\mathrm{WS}_{2}$ using the firstprinciples methods based on density functional theory. Co is typical ferromagnetic metal material, due to the interaction of Co atoms and the $\mathrm{WS}_{2}$ sheets. We need to consider the effect of the $\mathrm{WS}_{2}$ sheet on the Co atom magnetic sequence. We calculated the formation energy of the ferromagnetic (FM) and the antiferromagnetic (AFM) states under the same coverage, to illustrate the structures of Co-adsorbed monolayer $\mathrm{WS}_{2}$ which are ferromagnetic, ensuring the significance and feasibility of the simulation calculation.

\section{Methods}

All calculations were based on the density functional theory using the generalized gradient approximation (GGA) with Perdew-Burke-Ernzerhof (PBE) method. The electronic wave functions are described by the projector augmented wave (PAW) potential, as carried out in the Vienna $a b$ initio simulation package (VASP) [30]-[32]. The localdensity approximation (LDA) were selected as the exchange and correlation potential [33]-[35]. Although the DFT functional cannot describes very accurately all the characteristics of interactions, but the overestimate of the binding energy by LDA is almost 
compensated by the ignored van der Waals interactions [36]-[38]. A kinetic energy cutoff is set for the plane-wave expansion at $500 \mathrm{eV}$, the k-point meshes are generated by Gama-center Monkhorst-Pack scheme for integration over the first Brillouin zone [39]. As the Graphene-like $\mathrm{WS}_{2}$ is composed of a single layer of $\mathrm{W}$ and $\mathrm{S}$ atoms arranged in a two-dimensional (2D) honeycomb lattice, To detailedly explore the properties of Coadsorbed $2 \mathrm{D}$ systems $\left(\mathrm{Co} / \mathrm{WS}_{2}\right)$, a $(2 \times 2 \times 1) \mathrm{WS}_{2}$ super cell containing $4 \mathrm{~W}$ and $8 \mathrm{~S}$ atoms are modeled, a more than $20 \AA$ vacuum layer was set between two adjacent $\mathrm{WS}_{2}$ monolayers in the Z-direction to avoid inter-layer interactions. $(12 \times 12 \times 1)$ k-point meshes are adopted for the structural optimization, density of states, respectively. In each system, all the atomic positions were fully relaxed until the total energy was converged to less than $1 \times 10^{-7} \mathrm{eV} /$ atom and the forces on all atoms were below 0.01 $\mathrm{eV} / \AA$.

\section{Results and Discussions}

\subsection{The Adsorption of Co Atom}

According to the monolayer hexagonal lattice structure of $\mathrm{WS}_{2}$, we considered three basic types of adsorption sites (Figure 1): $\mathrm{T}_{\mathrm{W}}$ (top site directly above a $\mathrm{W}$ atom), $\mathrm{H}$ site (hollow site above the center of hexagons) and $\mathrm{T}_{\mathrm{S}}$ site (top site directly above a $\mathrm{S}$ atom). The height of adsorption is $3.5 \AA$, it is reasonable to expect the fully relaxation of Co adatom on one of these adsorption sites. In order to discuss the relative stabilities of the systems, the adsorption energy $\left(E_{\mathrm{a}}\right)$ is defined as:

$$
E_{\mathrm{a}}=E_{\mathrm{WS}_{2}}+E_{\mathrm{Co}}-E_{\mathrm{tot}}
$$

$E_{\text {tot }}$ is the total energy of $\mathrm{Co} / \mathrm{WS}_{2}$ system, $E_{\mathrm{WS}_{2}}$ is the total energy of the pure $\mathrm{WS}_{2}$ sheet and $E_{\mathrm{Co}}$ is the total energy of an isolated atom. After relaxation, the electron configurations, adsorption energies $E_{\mathrm{a}}$ and structural properties for a single Co adsorbed $\mathrm{WS}_{2}$ obtained from our calculations are listed in Table 1 . The adsorption energies of Tw- $\mathrm{WS}_{2}, \mathrm{H}-\mathrm{WS}_{2}$ and Ts- $\mathrm{WS}_{2}$ systems are $3.03 \mathrm{eV}, 2.48 \mathrm{eV}$ and $1.44 \mathrm{eV}$, respectively. They are all positive and the adsorption energies of $\mathrm{Tw}^{-} \mathrm{WS}_{2}$ is bigger than the others, which indicates the Co considered can chemically absorb on $\mathrm{WS}_{2}$ monolayer stably and $\mathrm{Tw}-\mathrm{WS}_{2}$ is the more stable structure.

(a)

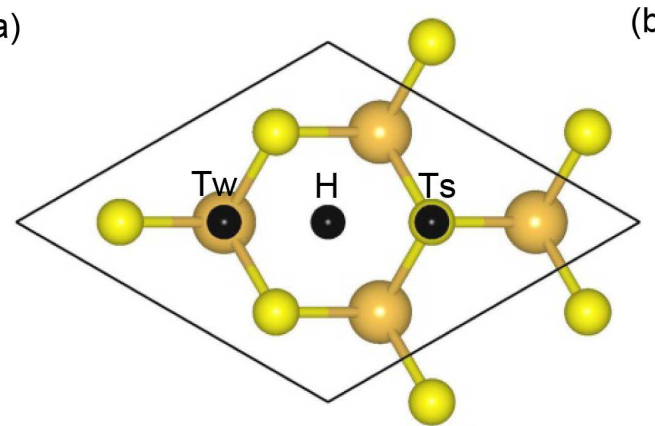

(b)

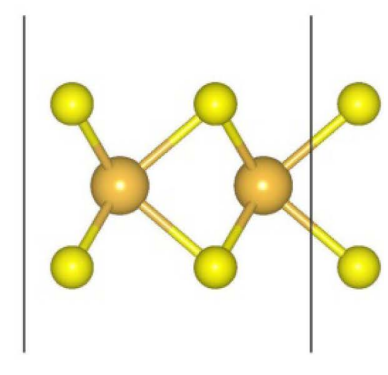

Figure 1. (a) (b) Top and side views of monolayer $\mathrm{WS}_{2}$, where yellow and orange balls stand for $\mathrm{S}$ atoms and $\mathrm{W}$ atoms, respectively. 


\subsection{The Influence of the Co-Adsorbed Monolayer $\mathrm{WS}_{2}$}

Among the different adsorption sites considered here, the bond lengths $d_{\mathrm{W}-\mathrm{s}}$ near the adsorption sites have been extended in different levels, the distortion introduced by adsorption can not be ignored, which are used to check the deformation of the $\mathrm{WS}_{2}$ monolayer. From Figure 2 and Table 1 , the $\mathrm{d}_{\mathrm{W}-\mathrm{s}}$ near the Tw adsorption position is $2.51 \AA$, compared with the pure $\mathrm{WS}_{2}(2.42 \AA), \mathrm{H}-\mathrm{WS}_{2}(2.48 \AA)$ and $\mathrm{Ts}-\mathrm{WS}_{2}(2.42 \AA)$ systems, The change is the most obvious. Suggesting that the interaction between Co adatom and the $\mathrm{WS}_{2}$ sheet is the most powerful, Tw site can be referred to as the favored site. Here, the corresponding vertical equilibrium height " $h$ " represents the distance between the adatom and $\mathrm{WS}_{2}$ hosts, it monotonically increases with increasing atomic size. Since the atom radius $\mathrm{r}_{\mathrm{S}}<\mathrm{r}_{\mathrm{Co}}<\mathrm{r}_{\mathrm{W}}$, so the "h" of the Tw-WS, $\mathrm{H}-\mathrm{WS} \mathrm{S}_{2}$ and Ts-WS $\mathrm{W}_{2}$ decreases successively. To illustrate the origin of the magnetic properties in $\mathrm{Tw}_{\mathrm{W}} \mathrm{WS}_{2}$ system, the total density of states (TDOS) and charge density difference are presented in Figure 3 and Figure 4, respectively.

TDOS of pure $\mathrm{WS}_{2}$ and Tw-WS $\mathrm{W}_{2}$ monolayers are shown in Figure 3, illustrating that the electronic properties of $\mathrm{WS}_{2}$ sheet is changed markedly upon the Co adatom. For isolated monolayer $\mathrm{WS}_{2}$, there is strong hybridization between the $\mathrm{W}$-d orbitals and the $\mathrm{p}$ orbitals of $\mathrm{S}$ atoms, leading a strong covalent bonding between $\mathrm{W}$ and $\mathrm{S}$, the sharing

Table 1. Calculated geometry of $\mathrm{T}_{\mathrm{W}}-\mathrm{WS}_{2}$ system, adsorption energy of adatom $E_{\mathrm{a}}$, total magnetic moment of the system $M_{\mathrm{tot}}$, local magnetic moment of the Co adatom $M_{\mathrm{Co}}$, W-S bond length $\mathrm{d}_{\mathrm{W}-\mathrm{S}}$, height of the adsorption $h$.

\begin{tabular}{cccccc}
\hline System & $E_{\mathrm{a}}(\mathrm{eV} /$ atom $)$ & $M_{\mathrm{tot}}\left(\mu_{\mathrm{B}}\right)$ & $M_{\mathrm{Co}}\left(\mu_{\mathrm{B}}\right)$ & $\mathrm{d}_{\mathrm{W}-\mathrm{S}}(\AA)$ & $\mathrm{h}(\AA)$ \\
\hline $\mathrm{WS}_{2}$ & - & - & - & 2.42 & - \\
$\mathrm{T}_{\mathrm{W}}$ & 3.03 & 1.00 & 0.94 & 2.51 & 2.56 \\
$\mathrm{H}$ & 2.48 & 1.00 & 0.95 & 2.48 & 2.36 \\
$\mathrm{~T}_{\mathrm{S}}$ & 1.44 & 1.00 & 1.37 & 2.42 & 2.03 \\
\hline
\end{tabular}

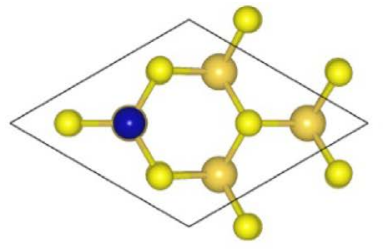

(1)

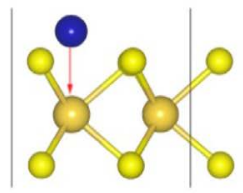

(1)

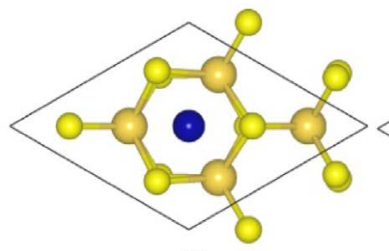

(2)

(a)

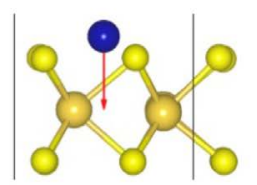

(2)

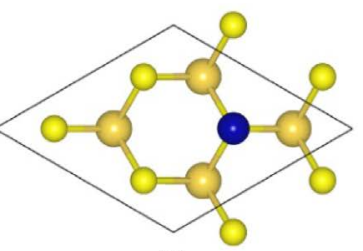

(3)

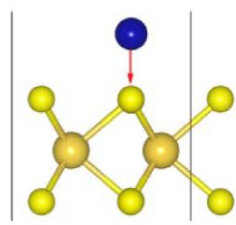

(3)

(b)

Figure 2. (1)-(3) depict the optimized structures of Co atom adsorption on monolayer $\mathrm{WS}_{2}$ sheet ((a): top view, (b): side view. blue balls stand for Co atoms). 

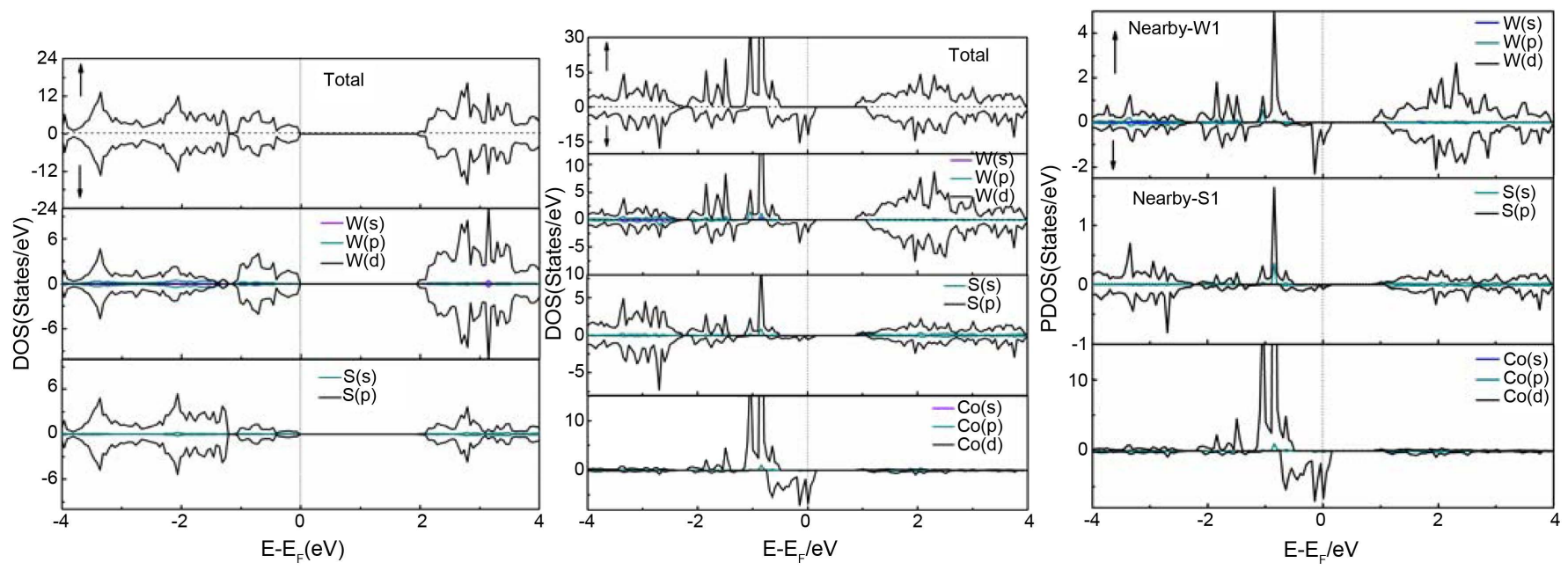

Figure 3. The total and partial density of states of clear monolayer $\mathrm{WS}_{2}$ and $\mathrm{Tw}-\mathrm{WS}_{2}$ system, the PDOS of Co and the nearby $\mathrm{W}$, $\mathrm{S}$ atom.
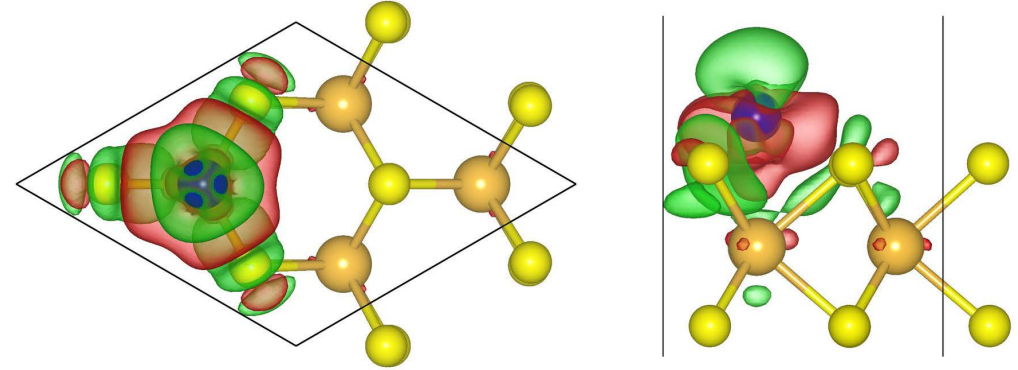

Figure 4. The electron charge density difference for $\mathrm{Tw}-\mathrm{WS}_{2}$ system, where red and green contours stand forgiven or loss electron density region, respectively.

degree of electronic states is high from $\sim-4.00$ to $\sim-1.00 \mathrm{eV}$, which forming a strong $\sigma$ bond; Near the Fermi level of the valence band, the contribution of the electronic states is more from the $\mathrm{W}$ atoms with a weaker $\pi$ bond formed, which is unstable. After a single Co adsorption, the band structure has been modified, especially near the Fermi level, where the conduction band as well as the valence band moves down. The Fermi level of $\mathrm{T}_{\mathrm{W}}$-WS $\mathrm{S}_{2}$ systems shift up more or less, which shows the impurity states leading from the Co adatom is very distinct in the original state of $\mathrm{WS}_{2}$ host. Since the occupation situation of the spin-up and spin-down states is very uneven, the spin-down states are full occupied while the spin-up states are not. The adsorption of transition metal Co atom induce a magnetic moment about $1.00 \mu_{\mathrm{B}}$ in $\mathrm{WS}_{2}$, which due to the strong hybridization between the $\mathrm{d}$ orbitals (came from the nearby $\mathrm{W}$ and Co atoms) and a little from the $\mathrm{p}$ orbitals (are from the nearby $\mathrm{S}$ atoms). Especially, the impurity states near the Fermi level mainly come from the Co atom.

To further explore the electronic distribution of $\mathrm{Tw}-\mathrm{WS}_{2}$ system, the charge density difference $(\Delta \rho)$ is determined by the following function:

$$
\Delta \rho=\rho_{\mathrm{Co} / \mathrm{WS}_{2}}-\rho_{\mathrm{WS}_{2}}-\rho_{\mathrm{Co}}
$$

$\rho_{\mathrm{Co} / \mathrm{WS}_{2}}, \rho_{\mathrm{WS}_{2}}$ and $\rho_{\mathrm{Co}}$ are the charge density of Tw- $-\mathrm{WS}_{2}, \mathrm{WS}_{2}$ sheet and isolated 
Co atom, respectively. It clearly shows that the charge density increases in the region between Co adatom and $\mathrm{W}$ or $\mathrm{S}$ atoms and causes the electron density of surrounding $\mathrm{S}$ atoms to loss. This suggests that all of $\mathrm{Co}, \mathrm{W}$ and $\mathrm{S}$ atoms interact with each other. These results are consistent with the analysis of the density of states, illustrating that there is a strong hybridization between Co adatom and the surface of the sheet.

\subsection{The Properties under the Different ML}

In order to further study the effect of Co adsorption to the properties of $\mathrm{WS}_{2}$ under the different monolayer coverage (ML). We investigate the electronic and magnetic properties of $\mathrm{Tw}-\mathrm{WS}_{2}$ in the $0.5 \mathrm{ML}$ and 1.0 ML (the number ratio of Co:W=1:2 and 1:1), as shown in Figure 5. Initially, we calculate the energy differences $(\Delta E)$ between the ferromagnetic ground state and the antiferromagnetic state at first, which is defined as following:

$$
\Delta E=\left[E_{\mathrm{AFM}}-E_{\mathrm{FM}}\right] / n
$$

$E_{\mathrm{FM}}$ and $E_{\mathrm{AFM}}$ are the total energy of the ferromagnetic ground state and the antiferromagnetic state under the same kind of monolayer coverage. From Table 2, the $\Delta E$ is positive, the total energy of the ferromagnetic ground state lower than that of the antiferromagnetic state. It is clear that the Co atoms preferentially display ferromagnetic coupling at both the $0.5 \mathrm{ML}$ and $1.00 \mathrm{ML}$.

Additional insight into the electronic structure of the Co-adsorbed $\mathrm{WS}_{2}$ monolayer can be obtained from the electronic total density of states (TDOS) displayed in Figure 6. For the $0.5 \mathrm{ML}$ and $1.0 \mathrm{ML}$, the TDOS shows the impurities state density wave peaks

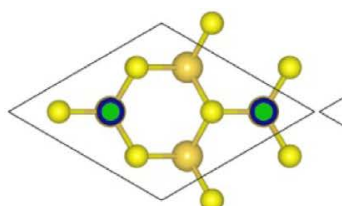

(1)

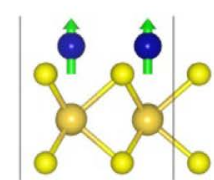

(3)

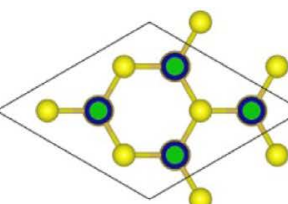

(2)

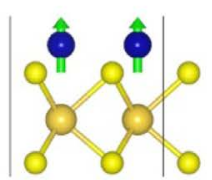

(4)

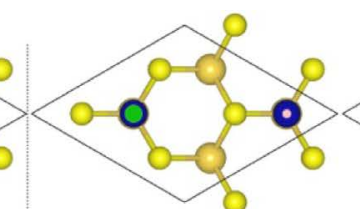

(5)

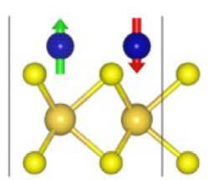

(7)

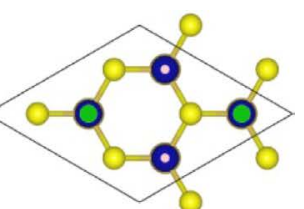

(6)

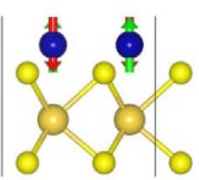

(8)

(a)

(b)

Figure 5. (a) (b) depict the structures of the ferromagnetic ground state and the antiferromagnetic state under the different Co ML (0.5 ML and 1.0 ML), where green and red contours represent spin-up and spin-down, respectively.

Table 2. Calculated geometry of $\mathrm{T}_{\mathrm{W}}-\mathrm{WS}$ system.

\begin{tabular}{cccc}
\hline System & $\Delta E(\mathrm{eV} /$ atom $)$ & $E_{\mathrm{a}}(\mathrm{eV} /$ atom $)$ & $M_{\mathrm{tot}}\left(\mu_{\mathrm{B}} /\right.$ atom $)$ \\
\hline $0.5 \mathrm{ML}$ & 0.103 & 3.39 & 1.00 \\
$1.0 \mathrm{ML}$ & 0.027 & 3.79 & 0.99 \\
\hline
\end{tabular}



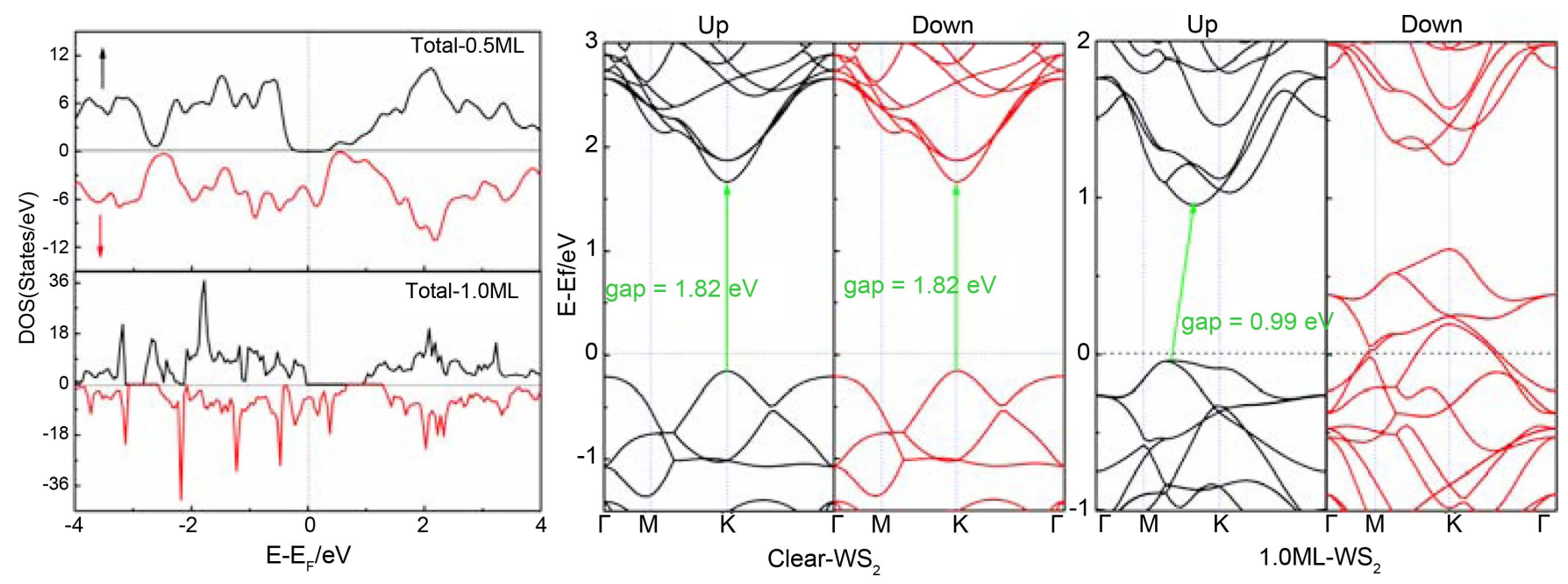

Figure 6. The total density of states under $0.5 \mathrm{ML}$ and 1.0 ML (left), the energy band diagram of clear monolayer WS and $^{2} .0 \mathrm{ML}^{2}$ system.

straddling the Fermi level in the spin down channels, while the spin up channels continues to display an appreciable gap, indicating that the doped monolayer is essentially half-metallic.

In order to illustrate the significant changes caused by Co adsorption in the nature of the monolayer $\mathrm{WS}_{2}$, we calculated the band structure of the pure monolayer $\mathrm{WS}_{2}$ and Tw-WS $\mathrm{W}_{2}$ systems (1.0 ML). Form Figure 6, It clearly shows that the band of the spin up and spin down channels are exactly the same, monolayer $\mathrm{WS}_{2}$ is a direct gap semiconductor, the valence band top and the conduction band bottom are located at the $\mathrm{K}$ point in the Brillouin zone. The gap is about $1.82 \mathrm{eV}$, which consistent with the experimental value and theoretical value [26] [27]. For 1.0 ML Tw-WS ture becomes a indirect band gap of $0.99 \mathrm{eV}$ semiconductor, while the spin down channel is characterized by the properties of the metal (Figure 6). Thus, it promises to be a compelling and feasible candidate-halfmetallic material for low-dimensional spintronic devices in the future.

\section{Conclusion}

We have performed first-principles electronic structure calculations to study the properties of Co-adsorbed monolayer $\mathrm{WS}_{2}$, and found that the $\mathrm{Tw}$ site is stable position of the Co adsorbed on the surface of the sheet, and the local magnetic moment is about $0.94 \mu_{\mathrm{B}}$ focused on the Co atomic adsorption. For the Tw-WS ${ }_{2}$, we further investigate the stability of the ferromagnetic (FM) and antiferromagnetic (AFM) states under the different monolayer coverage (ML). The calculation results of formation energy show that the ferromagnetic (FM) configurations are more stable at 0.50 ML and 1.0 ML. Both of the local electronic density states (LDOS) and band calculation indicate that two kinds of structure present the half-metal property.

\section{References}

[1] Butler, S.Z., Hollen, S.M., Cao, L., Cui, Y., et al. (2013) Progress, Challenges, and Oppor- 
tunities in Two-Dimensional Materials beyond Graphene. ACS Nano, 4, 2898-2926.

http://pubs.acs.org/doi/abs/10.1021/nn400280c

http://dx.doi.org/10.1021/nn400280c

[2] Chhowalla, M., Shin, S.H., Eda, G., Li, L.-J., Loh, K.P. and Zhang, H. (2013) The Chemistry of Two-Dimensional Layered Transition Metal Dichalcogenidenanosheets. Nature Nanotechnology, 5, 263-275.

http://www.nature.com/search?journal=nchem\&q=10.1038\%2FNCHEM.1589

[3] Liu, H., Han, H. and Zhao, J. (2015) Atomistic Insight into the Oxidation of Monolayer Transition Metal Dichalcogenides: From Structures to Electronic Properties. RSC Advances, 5, 17572-17572.

http://pubs.rsc.org/en/Content/ArticleLanding/RA/2015/C4RA17320A http://dx.doi.org/10.1039/C4RA17320A

[4] Yazyev, O.V. and Kis, A. (2014) $\mathrm{MoS}_{2}$ and Semiconductors in the Flatland. Materials Today, 18, 1369-7021.

[5] Ding, Y., Wang, Y., Ni, J., Shi, L., Shi, S. and Tang, W. (2011) First Principles Study of Structural, Vibrational and Electronic Properties of Graphene-Like $\mathrm{MX}_{2}(\mathrm{M}=\mathrm{Mo}, \mathrm{Nb}, \mathrm{W}$, Ta; X = S, Se, Te) Monolayers. Physica B, 406, 2254-2260.

https://www.researchgate.net/publication/229313422

http://dx.doi.org/10.1016/j.physb.2011.03.044

[6] Radisavljevic, B., Radenovic, A., Brivio, J., Giacometti, V. and Kis, A. (2011) Single-Layer $\mathrm{MoS}_{2}$ Transistors. Nature Nanotechnology, 6, 147-150.

https://www.researchgate.net/publication/49796080

http://dx.doi.org/10.1038/nnano.2010.279

[7] Wang, Q.H., Kis, A., Kourosh, K.-Z., Coleman, J.N. and Strano, M.S. (2012) Electronics and Optoelectronics of Two-Dimensional Transition Metal Dichalcogenides. Nature Nanotechnology, 7, 699-712. http://www.nature.com/doifinder/10.1038/nnano.2012.193 http://dx.doi.org/10.1038/nnano.2012.193

[8] Drescher, T., Niefind, F., Bensch, W. and Grünert, W. (2012) Sulfide Catalysis without Coordinatively Unsaturated Sites: Hydrogenation, Cis-Trans Isomerization, and $\mathrm{H}_{2} / \mathrm{D}_{2}$ Scrambling over $\mathrm{MoS}_{2}$ and $\mathrm{WS}_{2}$. Journal of the American Chemical Society, 134, 1889618899. http://dx.doi.org/10.1021/ja3074903

[9] Balog, R., et al. (2010) Bandgap Opening in Graphene Induced by Patterned Hydrogen Adsorption. Nature Materials, 9, 315-319. https://www.researchgate.net/publication/41944338 http://dx.doi.org/10.1038/nmat2710

[10] Shiva, K., Ramakrishna Matte, H.S.S., Rajendra, H.B., Bhattacharyya, A.J. and Rao, C.N.R. (2013) Employing Synergistic Interactions between Few-Layer $\mathrm{WS}_{2}$ and Reduced Graphene Oxide to Improve Lithium Storage, Cyclability and Ratecapability of Li-Ion Batteries. Nano Energy, 2, 787-793. http://dx.doi.org/10.1016/j.nanoen.2013.02.001

[11] Zhou, L., Yan, S., Pan, L., et al. (2016) A Scalable Sulfuration of WS $\mathrm{W}_{2}$ to Improve Cyclability and Capability of Lithium-Ion Batteries. Nano Research, 9, 857-865.

http://link.springer.com/article/10.1007/s12274-015-0966-9 http://dx.doi.org/10.1007/s12274-015-0966-9

[12] Yue, Q., Shao, Z., Chang, S. and Li, J. (2013) Adsorption of Gas Molecules on Monolayer $\mathrm{MoS}_{2}$ and Effect of Applied Electric Field. Nanoscale Research Letters, 8, 425.

http://link.springer.com/article/10.1186\%2F1556-276X-8-425 http://dx.doi.org/10.1186/1556-276X-8-425

[13] Rastogi, P., Kumar, S., Bhowmick, S., Agarwal, A. and Chauhan, Y.H. (2014) Doping Strategies for Monolayer $\mathrm{MoS}_{2}$ via Surface Adsorption: A Systematic Study. Journal of Physical 
Chemistry C, 118, 30309-30314. http://dx.doi.org/10.1021/jp510662n

[14] Ataca, C. and Ciraci, S. (2011) Functionalization of Single-Layer $\mathrm{MoS}_{2}$ Honeycomb Structures. Journal of Physical Chemistry C, 115, 13303-13311.

http://dx.doi.org/10.1021/jp2000442

[15] Dolui, K., Rungger, I., Pemmaraju, C.D. and Sanvito, S. (2013) Possible Doping Strategies for $\mathrm{MoS}_{2}$ Monolayers: An Ab Initio Study. Physical Review B, 88, Article ID: 075420. http://dx.doi.org/10.1103/PhysRevB.88.075420

[16] Rastogi, P., Kumar, S., Bhowmick, S., Agarwal, A. and Chauhan, Y.H. (2014) Doping Strategies for Monolayer $\mathrm{MoS}_{2}$ via Surface Adsorption: A Systematic Study. Journal of Physical Chemistry C, 118, 30309-30314. http://dx.doi.org/10.1021/jp510662n

[17] Chang, J., Larentis, S., Tutuc, E., Register, L.F. and Banerjee, S.K. (2014) Atomistic Simulation of the Electronic States of Adatoms in Monolayer $\mathrm{MoS}_{2}$. Applied Physics Letters, 104, Article ID: 141603. http://dx.doi.org/10.1063/1.4870767

[18] Li, X.D., Fang, Y.M., Wu, S.Q. and Zhu, Z.Z. (2015) Adsorption of Alkali, Alkaline-Earth, Simple and 3D Transition Metal, and Nonmetal Atoms on Monolayer MoS 2 . AIP Advances, 5, Article ID: 057143. http://dx.doi.org/10.1063/1.4921564

[19] Zhao, X., Xia, C., Wang, T. and Dai, X. (2016) Electronic and Magnetic Properties of $\mathrm{X}$-Doped $(\mathrm{X}=\mathrm{Ti}, \mathrm{Zr}, \mathrm{Hf})$ Tungsten Disulphide Monolayer. Journal of Alloys and Compounds, 654, 574-579. http://dx.doi.org/10.1016/j.jallcom.2015.09.160

[20] Menon, M. and Andriotis, A.N. (2014) Tunable Magnetic Properties of Transition Metal Doped $\mathrm{MoS}_{2}$. Physical Review B, 90, Article ID: 125304.

[21] Ramasubramaniam, A. (2013) Mn-Doped Monolayer $\mathrm{MoS}_{2}$ : An Atomically Thin Dilute Magnetic Semiconductor. Physical Review B, 87, Article ID: 195201. http://dx.doi.org/10.1103/physrevb.87.195201

[22] Matte, H.S.S.R., Gomathi, A., Manna, A.K., Late, D.J., Datta, R., Pati, S.K. and Rao, C.N.R. (2010) $\mathrm{MoS}_{2}$ and $\mathrm{WS}_{2}$ Analogues of Graphene. Angewandte Chemie International Edition, 49, 4059-4062. http://dx.doi.org/10.1002/anie.201000009

[23] Yang, J., Voiry, D., Ahn, S.J., Kang, D., Kim, A.Y., Chhowalla, M. and Shin, H.S. (2013) Two-Dimensional Hybrid Nanosheets of Tungsten Disulfide and Reduced Graphene Oxide as Catalysts for Enhanced Hydrogen Evolution. Angewandte Chemie International Edition, 52, 13751-13754. http://onlinelibrary.wiley.com/doi/10.1002/anie.201307475/full http://dx.doi.org/10.1002/anie.201307475

[24] Fang, X.P., Hua, C.X., Wu, C.R., Wang, X.F., Shen, L.Y., Kong, Q.Y., Wang, J.Z., Hu, Y.S., Wang, Z.X. and Chen, L.Q. (2013) Synthesis and Electrochemical Performance of Graphene-Like $\mathrm{WS}_{2}$. Chemistry, 19, 5694-5700. http://dx.doi.org/10.1002/chem.201204254

[25] Zhao, W.J., Ghorannevis, Z., Chu, L.Q., Toh, M.L., Kloc, C., Tan, P.H. and Eda, G. (2013) Evolution of Electronic Structure in Atomically Thin Sheets of $\mathrm{WS}_{2}$ and $\mathrm{WSe}_{2}$. ACS Nano, 7, 791-797. http://dx.doi.org/10.1021/nn305275h

[26] Zhao, X., Dai, X.Q., Xia, C.G., Wang, T.X. and Peng, Y.T. (2015) Electronic and Magnetic Properties of Mn-Doped Monolayer $\mathrm{WS}_{2}$. Solid State Communications, 215-216, 1-4. http://dx.doi.org/10.1016/j.ssc.2015.05.003

[27] Li, H., Liu, S., Huang, S., Yin, D., Li, C. and Wang, Z. (2016) Impurity-Induced Ferromagnetism and Metallicity of $\mathrm{WS}_{2}$ Monolayer. Ceramics International, 42, 2364-2369. http://dx.doi.org/10.1016/j.ceramint.2015.10.033

[28] Ma, D. and Yang, Z. (2011) First Principles Studies of Pb Doping in Graphene: Stability, Energy Gap, and Spin-Orbit Splitting. New Journal of Physics, 13, Article ID: 123018. http://dx.doi.org/10.1088/1367-2630/13/12/123018 
[29] Dai, X., Li, Y., Xie, M., Hu, G., Zhao, J. and Zhao, B. (2011) Structural Stability and Electronic, Magnetic Properties of Ge Adsorption on Defected Graphene: A First-Principles Study. Physica E, 43, 1461-1464. http://dx.doi.org/10.1016/j.physe.2011.04.006

[30] Kresse, G. and Hafner, J. (1993) Ab Initio Molecular Dynamics for Open-Shell Transition Metals. Physical Review B, 48, 13115-13118. http://dx.doi.org/10.1103/PhysRevB.48.13115

[31] Kresse, G. and Furthmüller, J. (1996) Efficiency of Ab-Initio Total Energy Calculations for Metals and Semiconductors Using a Plane-Wave Basis Set. Computational Materials Science, 6, 15-50. http://dx.doi.org/10.1016/0927-0256(96)00008-0

[32] Kresse, G. and Furthmüller, J. (1996) Efficient Iterative Schemes for ab initio Total-Energy Calculations Using a Plane-Wave Basis Set. Physical Review B, 54, 11169-11186. http://dx.doi.org/10.1103/PhysRevB.54.11169

[33] Perdew, J.P. and Zunger, A. (1981) Self-Interaction Correction to Density-Functional Approximations for Many-Electron Systems. Physical Review B, 23, 5048-5079. http://dx.doi.org/10.1103/PhysRevB.23.5048

[34] Okamoto, Y. and Miyamoto, Y. (2001) Ab Initio Investigation of Physisorption of Molecular Hydrogen on Planar and Curved Graphenes. Journal of Physical Chemistry B, 105, 3470-3474. http://dx.doi.org/10.1021/jp003435h

[35] Ao, Z., Jiang, Q., Zhang, R., Tan, T. and Li, S. (2009) Al Doped Graphene: A Promising Material for Hydrogen Storage at Room Temperature. Journal of Applied Physics, 105, Article ID: 074307. http://dx.doi.org/10.1063/1.3103327

[36] Zhao, S.J., Xue, J.M. and Kang, W. (2014) Gas Adsorption on $\mathrm{MoS}_{2}$ Monolayer from First-Principles Calculations. Chemical Physics Letters, 595-596, 35-42.

http://dx.doi.org/10.1016/j.cplett.2014.01.043

[37] Hu, A.M., Wang, L.L., Meng, B. and Xiao, W. (2015) Ab Initio Study of Magnetism in Nonmagnetic Metal Substituted Monolayer $\mathrm{MoS}_{2}$. Solid State Communications, 220, 67-71. http://dx.doi.org/10.1016/j.ssc.2015.07.011

[38] Perdew, J.P. and Zunger, A. (1981) Self-Interaction Correction to Density-Functional Approximations for Many-Electron Systems. Physical Review B, 23, 5048-5079. http://dx.doi.org/10.1103/PhysRevB.23.5048

[39] Monkhorst, H.J. and Pack, J.D. (1976) Special Points for Brillouin-Zone Integrations. Physical Review B, 13, 5188-5192. http://dx.doi.org/10.1103/PhysRevB.13.5188

\section{Submit or recommend next manuscript to SCIRP and we will provide best service for you:}

Accepting pre-submission inquiries through Email, Facebook, LinkedIn, Twitter, etc.

A wide selection of journals (inclusive of 9 subjects, more than 200 journals)

Providing 24-hour high-quality service

User-friendly online submission system

Fair and swift peer-review system

Efficient typesetting and proofreading procedure

Display of the result of downloads and visits, as well as the number of cited articles

Maximum dissemination of your research work

Submit your manuscript at: http://papersubmission.scirp.org/

Or contact msce@scirp.org 